\title{
Grammatische Kategorie oder kompositionaler ,Bedeutungseffekt'? Am Beispiel nicht-kanonischer Verknüpfungen mit sein
}

\section{Abstract}

The paper discusses the so called 'absentive'. It is shown that the absentive is not a grammatical category but a phenomenon with a conventionalised meaning that can be treated compositionally. We believe that the analysis proposed here must also be applied to the domain of information-structure.

\section{Zusammenfassung}

Ausgangspunkt in der (komparativen) Untersuchung sind nicht-kanonische Verknüpfungen mit sein im Deutschen, die durch das Muster NP+sein+Infinitiv (Martine ist spazieren) repräsentiert sind und in der Literatur mit dem Terminus Absentiv bezeichnet werden. Das Muster NP+sein+Infinitiv - so wird behauptet - weise eine spezifische Bedeutung auf, die in anderen Fällen bspw. durch den Ausdruck weg explizit gemacht werden (Martine ist weg spazieren), aber im sog. Absentiv gerade ohne overte Determination bleibt.

Geprägt hat den Terminus Absentiv De Groot (2000). Der Gegenstand selbst ist inzwischen in vielen Arbeiten aufgegriffen worden (cf. Abraham 2007, Haslinger 2007, Vogel 2007, 2009). Mit wenigen Ausnahmen wird seit De Groot - so auch in fast allen darauf folgenden Arbeiten - die Auffassung vertreten, dass es sich beim Absentiv um eine (universelle) grammatische Kategorie handle, der als Komplex eine Abwesenheitsbedeutung (als Konstruktionsbedeutung) zugeschrieben wird. ${ }^{1}$ Im Deutschen insbesondere läge der Absentiv als „Vollkatego-

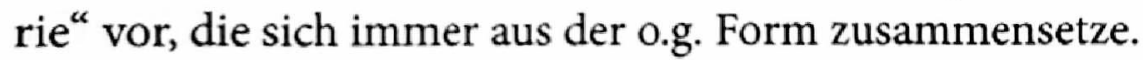

Die folgende komparative Untersuchung zum Deutschen, Französischen und Italienischen widerspricht der Sichtweise einer grammatischen Kategorie für

1 Postuliert wird eine grammatische (Teil-)Kategorie (wie für das Französische). Ähnliche Formeinheiten/Muster sind über Sprachen hinweg vertreten: Niederländisch, Deutsch, Ungarisch (Kopula+Infinitiv), Faröisch (Kopula+zu/to-Infinitiv), Norwegisch, Schwedisch (Kopula+und+Finitum), Italienisch (Kopula+lokative Präposition+Infinitiv), vgl. De Groot (2000: 696-713) und Vogel (2007: 266ff.). 
den Absentiv und skizziert eine Analyse, aus der sich Struktur und Bedeutung für das Deutsche kompositional ableiten lassen, die auch dem Sprachvergleich Stand hält, bzw. durch den Sprachvergleich Unterstützung erfährt. Unter Betrachtung einer Reihe von syntaktischen und interpretativen Eigenschaften der Instanziierungen des sog. Absentivs im Deutschen und den Vergleichssprachen, wird weiter gezeigt, dass einige empirische Behauptungen nicht ausreichend motiviert sind - speziell zum Französischen mit einer sog. Teilkategorie Absentiv. Auf der Grundlage der Finalitätshypothese wird dann auch die Abwesenheitsinterpretation als durch eine Implikatur hervorgerufen erschlossen (cf. Fortmann/Wöllstein 2013, Wöllstein 2013). Der Blick auf Daten in den romanischen Sprachen Italienisch und Französisch zeigt darüber hinaus, dass die Möglichkeiten der Realisierung der beteiligten grammatischen Komponenten übereinzelsprachlich unterschiedlich beschränkt sind.

Im Beitrag wird auch und gerade mit Blick auf informationsstrukturelle Phänomene dafür plädiert, von Verkürzungen abzusehen und weder Phänomene durch Konstruktionsbedeutung zu interpretieren, statt sie konventionalisierten Lesarten zuzuordnen, noch eine Konstruktionsbedeutung direkt grammatischen Einheiten zuzuweisen, ohne dass die Strukturen ausführlichen grammatischen Untersuchungen unterzogen werden.

\section{Worum es geht, Forschungsannahmen und Hypothese}

Alle (kontrastiven) Arbeiten gehen davon aus, dass mit dem sog. Absentiv (1a-c) eine durch lexikalische oder konstruktionell bedingte Eigenschaft zugewiesene Abwesenheitsbedeutung vorliegt und schlagen je nach theoretischer Perspektive unterschiedliche Lexikonerweiterungen vor (ausgenommen Haslinger 2007): ${ }^{2}$

a. Erst vergangenen Samstag war Monika Kruse aus. Hat sich amüsiert, hat gefeiert und geschwitzt. Monika Kruse war tanzen. Selbstverständlich ist das nicht.

T08/SEP.03958 die tageszeitung, 25.09.2008, S. 28; Zu gelockt für den Club

2 Folgende Ansätze sind in Fortmann/Wöllstein (2013, 2014) i.E. erläutert: Verbalprädikation mit Konstruktionsbedeutung: u.a. De Groot (2000), Ebert (1996), Krause (2002); Bewegungsverb-Ellipse: u. a. Abraham (2007); Lexikalische sein-Varianten (verschiedener Ausprägung): Haslinger (2007), König (2009), Vogel (2009); am als intrinsischer Topikort: Wöllstein $(2005,2009)$, Wöllstein/Rapp (2007). Alle vertreten eine Sichtweise für die Anreicherung entweder a) einer lückenhaften/elliptischen Struktur, oder b) des Lexikons. 
b. Auch hier ist der Vorbericht meist typisch - der Hund war spazieren und hat plötzlich anfallsartig zu niesen begonnen. Häufig wird ein Auge zugekniffen und die Nase beim Niesen nach einer Seite verzogen, oftmals kann man einseitiges, leichtes Nasenbluten feststellen.

NON09/JUL.12868 Niederösterreichische Nachrichten, 28.07.2009,

S. 10; Vorsicht bei Schliefhanseln

c. Bei Cinderella denkt man eigentlich mehr an Prinzen, aber Cinderella muss das Zimmer aufräumen. Obwohl sie eigentlich lieber lesen würde. Was auch ziemlich öde ist, wie Cinderellas aufgedrehte Schwester Clorinda findet. Mama ist shoppen, die Schwestern fetzen sich und Papa ist wieder mal total überfordert. Na ja, Familie eben.

B03/MAR.16158 Berliner Zeitung, 08.03.2003; Aschenputtel und der Superstar [S. 7]

Es wird auch nicht angezweifelt, dass der sog. Absentiv als (einzige) Antwort auf die wo-Frage den entscheidenden Hinweis auf ihre spezifische „Bedeutung“ belegt (2a); dass hingegen (2b) eine ebenso angemessene Antwort ist, bleibt unbeachtet: ${ }^{3}$

Wo ist Martine?

a. Martine ist lehren/einkaufen/...

b. Martine lehrt/kauft ein/...

Strukturell entsprechend schlicht (3a, b) - aufgrund der vermeintlich poor syntax (Abraham 2007) des sog. Absentiv -, scheint er weder informationsstrukturell verwertbar bzgl. Art und Weise der Informationsdarstellung und als Struktur mit Konstruktionsbedeutung untauglich für die systematische Ermittlung bspw. der Topik-Kommentar-Struktur noch an den Schnittstellen Morphologie/ Syntax/Semantik einer Exploration zugänglich:

a. Martine ist spazieren.

b. [einfacher Satz NP sein reiner Infinitiv]

Entgegen des De Grootschen Ansatzes fassen Fortmann/Wöllstein (2013) und Wöllstein (2013) den sog. Absentiv nicht als eigenständige grammatische

3 Die angeblich (ausschließlich) mit dem sog. Absentiv verbundenen interpretatorischen Eigenschaften, die aus der Absenz resultieren, einschließlich des deiktischen Grundkonzepts aus dem Absenz abgeleitet wird, halten weder wahrheitsfunktionalen Betrachtungen noch semantischer Fundierung stand, s. Fortmann/Wöllstein (2013: 78-81) und ausführlich in Fortmann/Wöllstein (2014). 
Kategorie auf, die Ausdruck von Abwesenheit des Subjektreferenten ist (4), sondern als komplexe kompositional zu ermittelnde Satzstruktur (5a) mit einer aus den regulären grammatischen Mitteln resultierenden Semantik (5b), auf dessen Basis die Abwesenheit des Subjektreferenten dann erst qua Implikatur erschlossen wird (5c). Damit ist der sog. Absentiv weder lückenhaft/elliptisch oder stumm, sondern syntaktisch reicher als die Oberfläche vermuten lässt.

(4) Martine ist weg spazieren.

a. [CP Martine ist $\mathrm{e}_{\text {LOC }}$ [VP spazieren]].

b. Semantik: Martine ist am Ort X, (um) Y (zu) tun.

c. Wenn Martine $Y$ nicht tut, ist sie am erwartbaren Ort; sonst abwesend.

Der sog. Absentiv im Deutschen weist zwar eine Unterbestimmtheit der Form-/ Funktionsbeziehung auf (bildet in gewisser Weise einen isolierten Typ), die aber nur auf den ersten Blick eine Bedeutungskonstitution auf Basis der beteiligten grammatischen Einheiten und ihrer regulären syntaktischen Verknüpfung bezweifeln lässt; es wird i.F. gezeigt, dass die Gesamtstruktur auf der Basis regulärer grammatischer Bestandteile zu analysieren und damit dann auch informationsstrukturell verwertbar ist, um dem augenblicklichen Interesse und dem Informationsstand des Adressaten gerecht zu werden (Halliday 1967).

Neben den Strukturen ohne (overten) Lokationsausdruck lassen sich auch Strukturen mit (overtem) Lokationsausdruck bilden (6), vgl. auch die Daten aus dem DeReKo (7); ohne eine nähere triftige Begründung von De Groot (2000) wie in der gesamten Literatur zum Thema sorgt das Nichtbeachten dieser Strukturen dafür, dass eine womöglich parallele kompositionale Analyse von vornherein ausgeschlossen wird, obwohl jede in die andere umwandelbar ist, ohne die Grammatikalität zu beschränken:

(6) Martine ist am Strand spazieren.

(7) a. Wir haben viel gespielt, waren im Meer schwimmen und haben die Insel ausführlich erkundet. Interessant und lehrreich war auch die Wattwanderung. Ins Watt kann man nur bei Ebbe gehen. Dort gibt es Krebse, Krabben, Muscheln und sogar einige Feuerquallen. Unsere Lehrerin hat Wattwürmer mit einem Spaten ausgegraben. An sehr feuchten Stellen muss man aufpassen, dass man nicht zu tief einsinkt.

BRZ05/OKT.04002 Braunschweiger Zeitung, 05.10.2005; Krebse,

Krabben, Feuerquallen: Im Watt gibt es viel zu entdecken

b. Zu den dramatischsten Brand-Einsätzen, die die Freiwillige Feuerwehr im Bezirk bewältigen musste, zählten vergangenes Jahr ein Dachstuhlbrand in Matzen, wo neun Atemschutztrupps das Feuer bekämpften 
und nach der Hausbesitzerin suchten. Die Menschenrettung konnte aber glücklicherweise abgebrochen werden, die Frau war in Gänserndorf einkaufen.

NON09/AUG.08749 Niederösterreichische Nachrichten,

18.08.2009, S. 7; Über 250.000 Euro mehr Brandschäden als 2007!

c. Das war keine Bedrohung im militärischen Sinn. Auf dem Golan war ich joggen, als plötzlich ein Rudel syrischer Hunde hinter mir her hetzte. Ich war unbewaffnet, war ja im Jogginganzug. Da ist mir eingefallen, was die Syrer in so einem Fall machen: Ich habe mit Steinen nach den Hunden geworfen, das hat sie Gott sei Dank vertrieben.

NON07/MAR.08128 Niederösterreichische Nachrichten,

14.03.2007, S. 31; „Sind da, um zu helfen und Präsenz zu zeigen“

d. Das Hobl \& Scher steht seit Dezember 1997 für Ess- und Trinkkultur in Wiener Neustadt. Ein gutes Glaserl Rotwein war auch die Basis für den Verkauf des Lokales. Manfred Schermann wollte sein Restaurant schon länger verkaufen. Mikulasek: „Ich war hier Wein einkaufen, bei einem Achterl haben wir über Gastronomie philosophiert."

BVZ09/MAR.01258 Burgenländische Volkszeitung,

11.03.2009,S. 28; Kurt Mikulasek neuer Boss von „Hob \& Scher“

Das ansonsten gleichbleibende Muster zu (5) fügt sich hier auf natürliche Weise ein:

a. [CP Martine ist am Strand ${ }_{L O C}$ [VP spazieren]].

b. Semantik: Martine ist am Strand, (um) Y (zu) tun.

Den Ausgangspunkt der Analyse hier, der den Strukturen (5) und (8) zugrunde liegt, bildet die Finalitätshypothese FH0 mit ihren strukturellen Implikationen FH1 und FH2 (Wöllstein 2013: 187f.):

(9) Finalitätshypothese (FH):

(FH0) Prädikativkonstruktionen des Typs in den Beispielen (1) und (3a) bilden eine Prädikativkonstruktion lokaler Ausprägung und semantisch finalem Adjunkt.

(FH1) Der Bezugssatz (=Prädikativkonstruktion) weist immer eine coverte oder overte Lokationsprädikation auf, vgl. (5a) und (6).

(FH2) Syntaktisch handelt es sich bei Bezugssatz um eine Satzprojektion $(\mathrm{CP})$ und bei der eingebetteten Struktur um eine Verbprojektion (VP), vgl. (8a). 
Folgende basale Formeinheiten können für das Deutsche identifiziert werden: Subjekt-NP, Form von sein, infinite Verbform im 1. Status (V-en). Den Formeinheiten "NP + sein + Infinitiv" folgend und der Einfachheit geschuldet wird i.F. die Rede von der „sein+Infinitiv-Struktur“ sein (nicht vom sog. Absentiv).

Folgende Abschnitte fundieren unmittelbar die Finalitätshypothese indem sie aufzeigen a) welche Beschränkungen die identifizierten Formeinheiten aufweisen, b) wie durch die Fokus-Hintergrundgliederung der „sein+Infinitiv-Struktur" der Rückschluss auf die syntaktische Struktur ermöglicht wird und c) dass die gegenseitigen Beschränkungen von a) und diachrone Befunde die Semantik der Struktur aufdecken helfen. ${ }^{4}$

\section{Grammatische Eigenschaften}

\subsection{Semantische und kategoriale Beschränkungen}

Die sein+Infinitiv-Struktur weist spezifische Beschränkungen bei der Wahl des Subjekts (10a, b) und des Infinitivs (11) auf. Diese Beschränkungen begegnen uns sowohl bei overter wie bei coverter Lokationsprädikation (übrigens gilt Gleiches nicht für um-zu, s. (34)-(39)); (10c, d) illustriert, dass keine grundsätzliche Unverträglichkeit zwischen Subjekt, Lokation und Verb für die Ungrammatikalität in (10a, b) und (11) verantwortlich sein kann.

Subjekt-Beschränkungen:

(10) a. ${ }^{*}$ Der Ofen ist (im Zimmer) uns einheizen.

b. ${ }^{\star}$ Der Keil ist das Holz spalten.

c. Der Ofen ist im Zimmer.

d. Der Ofen heizt uns ein./Der Keil spaltet das Holz.

Infinitiv-Beschränkungen:

(11) a. *Alex ist (im Wald) sich vor Geistern fürchten.

b. *Alex ist (im Bett) aufwachen/Geschenke kriegen.

c. ${ }^{*}$ Alex ist (im Wald) dem Fuchs begegnen.

Die Daten in (10a, b) und (11) zeigen, dass die sein+Infinitiv-Struktur agentive/intentionale Verben verlangt und referenzielle intentionale Subjekte (1), (7), marginal genügt auch eine (zweckgerichete) (Selbst-)Tätigkeit (12):

4 In den folgenden Abschnitten werden Beispiele aufgegriffen, die in Arbeiten von Christian Fortmann und mir (gemeinsam) bereits veröffentlicht sind und die hier nicht i.E. durch Zitierung der Beispielnummern gekennzeichnet sind. 
(12) a. ?Der Trecker ist Furchen ziehen.

b. Alex ist sich die Haare schneiden.

In Verbindung mit Vollverben weist der infinite Status auf den kategorialen Status von sein als Kopulaverb hin, da a) das Auxiliar sein ausschließlich den 3. Status ergativer Verben regiert (13a, b) vs. (13c) und die Kopula sein im Deutschen nie den reinen Infinitiv subkategorisiert; sie regiert b) den 3. Status in Prädikativkonstruktionen (14) und weist nicht die Intentionalitäts-Beschränkung von (10), (11) auf; in modaler Verwendung (15) regiert sie den 2. Status (und hier gilt die umgekehrte Beschränkung auf nichtdesignierte/-intentionale Subjekte (15a), bzgl. intentionaler Verben hier unbeschränkt $(15 b, c)$.

(13) a. Alex ist/war gelaufen.

b. *Alex bleibt gelaufen.

c. Alex *ist/hat Holz gehackt.

(14) a. Alex ist gefürchtet.

b. Der Schlüssel ist gefunden.

(15) a. *Alex ist zu laufen.

b. Alex ist/bleibt zu fürchten/zu suchen.

c. Holz ist/bleibt zu hacken.

Da sein/bleiben i.d.R. keine VP regiert und weder sein-Auxiliar noch sein-Kopula den 1. Status regieren, weist dennoch die Substitution von sein durch bleiben auf die Kopula hin, unabhängig von der Rektion:

(16) a. Alex ist $\left[_{\mathrm{VP}}\right.$ noch ein bisschen Holz hacken] geblieben.

b. Ich bleibe noch ein bisschen $\left[_{\mathrm{vP}}\right.$ Unkraut zupfen].

\section{Zwischenfazit:}

Es handelt sich

1. um eine Prädikativkonstruktion; die aber mit nichtkanonischem Infinitiv auftritt, weshalb

2. der Infinitiv - nehmen wir o.g. ernst - nicht den Status eines Komplements sondern eines Adjunkts aufweist (s. auch (17a)); zudem

3. liegt eine intentionale Semantik vor - qua modalem 1. Status (Reis 1995, Rapp/Wöllstein 2013)

Strukturell können die Verhältnisse in (5) wie folgt präzisiert werden: a) es handelt sich um eine Prädikativkonstruktion mit Kopula sein b) das prädikative Subjekt ist semantisch intentional und das Vollverb im 1. Status muss c) intentional sein, ist aber d) nicht subkategorisiert und damit nicht prädikatives Komplement. 
Folgende Fragen schließen unmittelbar an: Um welchen Typ Prädikation handelt es sich? M.a.W.: Was ist das prädikative Komplement (17a) und welche Adjunkte weisen intentionale Semantik auf (17b)?
a. $\left[_{\mathrm{CP}}\right.$ Subjekt $_{\mathrm{NP}}$ [ Kopula [ ${ }_{\mathrm{XP}}$ prädikatives Komplement $\left.\left.]\right]\right]$
b. $\left[{ }_{\mathrm{CP}}\right.$ Prädikativkonstruktion $\left[{ }_{\mathrm{XP}}\right.$ Adjunkt $\left.]\right]$

\subsection{Strukturelle Voraussetzungen}

Die vergleichende Untersuchung der folgenden Strukturtypen in (18) und (19) ist dadurch gerechtfertigt, dass jede Form mit overtem Lokationsausdruck in eine ohne einen solchen umgeformt werden kann und umgekehrt - ohne Einschränkung der Grammatikalität. Die Rechtfertigung - zumindest für das Deutsche - ergibt sich aus übereinstimmenden Vorkommensbeschränkungen in (10a) und (11). Es existiert eine Parallel-Konstruktion, die sich einzig durch das Vorkommen eines phonologisch overten Lokationsausdrucks unterscheidet:

(18) a. Martine ist spazieren/lehren.

b. Alex ist Holz hacken.

a. Martine ist am Strand/in der Uni lehren.

b. Alex ist im Hof Holz hacken.

Dass von multipler Prädikation auch mit dem Lokationsausdruck einerseits und dem Infinitiv andererseits nicht ausgegangen werden kann, zeigt der generelle Ausschluss multipler Prädikation in (20):

(20) a. ${ }^{*}$ Theo ist ein Idiot unverschämt.

b. *Der Tisch ist aus Buchenholz schön.

c. ${ }^{\star}$ Der Wein ist im Keller kühl.

Eine gültige Interpretation von (20c), die grammatisch ist, ist keine unter doppelter Prädikation. (20c) erlaubt natürlich eine Lesart, bei der die PP ein sekundäres Prädikat neben kühl darstellt, vgl. Der Wein ist im Keller bei $12^{\circ} \mathrm{im}$ Fass kühl.

\subsection{Prädikationstyp}

Erste Hinweise auf den möglichen Prädikationstyp gibt die overte Realisierung lexikalischen Materials mit NP, AP oder PP (21a). Aber nur eine PP kann im Kontext der sein+Infinitiv-Struktur auftreten (21b, c):

(21) a. Alex ist Maurer/blond/im Hof.

b. Alex ist ${ }^{*}$ Maurer $/{ }^{*}$ blond/im Hof arbeiten.

c. Alex ist im Hof arbeiten/im Wald Holz hacken. 
Zwischenfazit:

1. Bei in (21b) gegebener Gesamtform kommt NUR die Lokation als prädikatives Komplement in Betracht (21b)', womit FH1 bestätigt ist.

2. Ein erster Hinweis auf eine zweigliedrige Struktur ist gegeben.

Für die Zweigliedrigkeit spricht neben (21b), dass neben einer, s. (22a), immer auch zwei Intonationsphrasen bei der sein+Infinitiv-Struktur vorliegen können, s. (22b). Das strukturelle Äquivalent dazu bilden jeweils (23a) und (23b).

a. Alex ist im /Hof HOLZ hacken

b. Alex ist im /HOF / HOLZ hacken

a. Theo ist [ $\mathrm{vp}_{\mathrm{p}}$ im Hof Holz hacken]

b. Theo ist im Hof $\left[_{\mathrm{VP}}\right.$ Holz hacken]

Für die lokative Prädikativkonstruktion (=Lokationsprädikat als prädikatives Komplement) sprechen weiter, dass a) von der vollen lokalen Explizierung (24a) stufenweise eine ohne lokale Spezifikation in der VP (24b), eine indefinite (24c) und die coverte ( $24 \mathrm{~d}, \mathrm{e}$ ) möglich sind und dass b) auf die coverte Lokation bspw. mit dort referiert werden kann (25a) nicht aber auf eine, in der die Lokation nicht obligatorischer Bestandteil ist (25b).

(24) a. Eddi ist in LemWERder [ ${ }_{\mathrm{vP}}$ auf der WERFT arbeiten]

b. Eddi ist in Lemwerder $\left[_{\mathrm{VP}}\right.$ arbeiten]

c. Eddi ist irgendwo [ ${ }_{\mathrm{vp}}$ arbeiten]

d. Eddi ist $e_{\mathrm{LOC}}[\mathrm{VP}$ auf der Werft arbeiten]

e. Eddi ist $e_{\mathrm{LOC}}[\mathrm{VP}$ arbeiten]

(25) a. Theo ist Kohlen holen. Aber er wird nicht lange dort bleiben.

b. Theo holt Kohlen. \#Aber er wird nicht lange dort bleiben.

Fazit $z u \mathrm{e}_{\mathrm{LOC}}$

Die Lokation (LOC) ist bei stummer Prädikation $\boldsymbol{e}$ der grammatische Default.

\section{Syntaktische Struktur}

Dass es sich beim finalen infiniten Adjunkt auch nicht um eine CP handelt (sondern um eine VP, deren semantischer Typ einen Lokationsausdruck lizenzieren kann), darauf weist externe Evidenz hin: Der 1. Status kann nicht höher projizieren als VP; konsequenterweise sind innerhalb der Projektion nur Quantoren und Pluralia, nicht aber (identifizierende) Individualausdrücke möglich. Damit ist $\mathrm{FH} 2$ bestätigt. 
(26) a. Wir sind/waren in Freiburg, alle/jeder Martine gratulieren.

b. ${ }^{*}$ Wir sind/waren in Berlin, die Linguisten Martine gratulieren.

Wir beobachten, dass unter der stummen/coverten Lokation - trotz Zweigliedrigkeit der Struktur (27a) - keine Nachstellung des VP-Adjunkts möglich ist (27b):

a. dass Theo am Strand ist [einen Hornhecht angeln]

b. *dass Theo ist [einen Hornhecht angeln]

Die Ungrammatikalität von (27b) ist ein zusätzliches Indiz für die Lokation als Default, weil speziell leere Kategorien in der Grammatik einer Lizenzierung bedürfen. Genau dies ist unter Nachstellung des Infinitivs in (27b) nicht mehr gegeben. Durch die Nachstellung ändert sich der Adjunktionsort der infinitivischen VP und damit die relative Situierung zum leeren Lokationselement, s. dazu auch Fortmann/Wöllstein (2013: 90).
a. $\left[{ }_{\mathrm{CP}} \ldots\left[_{\mathrm{IP}} \mathrm{NP} \ldots\left[e_{\mathrm{LOC}}\left[\left[_{\mathrm{VP}} \ldots \mathrm{V}_{-\mathrm{FIN}}\right] \operatorname{sein}_{+\mathrm{FIN}}\right]\right]\right]\right]$
b. $\left[{ }_{\mathrm{CP}} \ldots\left[_{\mathrm{IP}} \mathrm{NP} \ldots\left[\mathrm{PP}\left[\left[_{\mathrm{VP}} \ldots \mathrm{V}_{\text {-FIN }}\right] \operatorname{sein}_{+\mathrm{FIN}}\right]\right]\right]\right]$
a. $\left[\mathrm{CP} \ldots\left[\left[_{\mathrm{IP}} \mathrm{NP} \ldots\left[e_{\mathrm{LOC}}\left[\operatorname{sein}_{+\mathrm{FIN}}\right]\right]\right]\left[\mathrm{VP}_{\mathrm{VP}} \ldots \mathrm{V}_{\text {-FIN }}\right]\right]\right]$
b. $\left[\left[_{\mathrm{CP}} \ldots\left[\left[_{\mathrm{IP}} \mathrm{NP} \ldots\left[\mathrm{PP}\left[\operatorname{sein}_{\mathrm{FIN}}\right]\right]\right]\left[_{\mathrm{VP}} \ldots \mathrm{V}_{\text {-FIN }}\right]\right]\right]\right.$

In der Strukturvariante (28a) ist die infinitivische VP innerhalb der VP des Matrixsatzes adjungiert. Bei Nachstellung kann nur an eine Projektion des Finitums (als IP bezeichnet) adjungiert werden. Die Lizenzierung leerer Kategorien erfordert allgemein eine enge lokale Nachbarschaft von lizenzierendem und lizenziertem Element; (27a) lässt sich mit der Struktur (29a) qua nicht erforderlicher struktureller Nähe erklären.

Dass keine gegangen-Ellipse vorliegt (wie in Abraham 2007 vorgeschlagen), zeigt sich daran, dass die sein+Infinitiv-Struktur nicht nur mit direktionalen Lokationsausdrücken verträglich ist:

(30) a. Alex ist *im Hof/in den Hof gegangen, Holz hacken.

b. Alex ist *im Hof/in den Hof Holz hacken gegangen.

Damit steht die Grundstruktur fest: sie ist zweigliedrig, wenn auch das Zweitglied nicht wie infinite satzwertig Adjunkte uneingeschränkt nachgestellt werden kann, s. (28a) wiederholt als (31).

$$
\left[{ }_{\mathrm{CP}} \ldots\left[_{\mathrm{IP}} \mathrm{NP} \ldots\left[e_{\mathrm{LOC} / \mathrm{PP}}\left[\left[_{\mathrm{VP}} \ldots \mathrm{V}_{\text {-FIN }}\right] \operatorname{sein}_{+\mathrm{FIN}}\right]\right]\right]\right]
$$

Welche semantische Ausprägung dem infiniten Adjunkt zukommt, wird im nächsten Abschnitt behandelt. 


\section{Formale Verwandtschaften}

Hinweise auf formale Verwandtschaften kann overt realisiertes lexikalisches Material geben, das auf den Typ des Adjunkts hinweist, vgl. (17b) wiederholt als (32).

$$
\left[_{\mathrm{CP}} \text { Prädikativkonstruktion }\left[{ }_{\mathrm{XP}} \text { Adjunkt }\right]\right]
$$

Im Deutschen werden lexikalisch infinite finale Adjunkte durch $u m-z u$ ausgedrückt; gleichzeitig verfügen finale Adjunkte über eine intentionale Semantik - synchron und diachron. In den indoeuropäischen Sprachen wurden bspw. Richtung, Ziel und Zweck durch Kasusmarker (Dativ, Akkusativ und Lokativ) ausgedrückt (Haspelmath 1989: 291ff.). Typologisch hat auch das Deutsche bei der Grammatikalisierung von Ziel bzw. Zweck (= Finalität) einen universalen Grammatikalisierungspfad von purposiven Elementen zu Infinitiven (im Dt. reiner Infinitiv) vollzogen. Wir können von einer lexikalischen Substitution der Finalitätsmarkierung bis zum GwD sprechen: ${ }^{5}$

$$
\begin{aligned}
& \text { reiner Inf. } \rightarrow \quad z u \text {-Infinitiv } \rightarrow \quad u m z u \text {-Infinitiv } \\
& -e n \quad \rightarrow \quad z u+-e n \rightarrow \quad u m+z u+-e n
\end{aligned}
$$

Mit der lexikalischen Substitution der Finalitätsmarkierung geht allerdings synchron eine grammatische semantische Beschränkung einher, die darin besteht, dass in der Prädikativkonstruktion der reine Infinitiv Finalität grammatisch und nicht lexikalisch ausdrücken kann. D. h. grammatischen Ausdruck findet Finalität in der Beschränkung auf die intentionale Semantik beim prädikativen Subjekt und beim reinen Infinitiv.

(keine) Subjekt-Beschränkung bei um-zu

(34) a. ${ }^{\star}$ Der Ofen ist (im Zimmer), uns einheizen/einzuheizen.

b. Der Ofen ist im Zimmer, um uns einzuheizen.

c. ${ }^{*}$ Der Keil ist (im Stamm), das Holz spalten/zu spalten.

d. Der Keil ist im Stamm, um das Holz zu spalten.

5 Vgl. dazu Wöllstein (2013: 189): „Diachron ist dabei im Deutschen jedoch nicht erst der Marker ahd. $z i$ oder mhd. $z u$ oder aber Formen der Präposition umb/umbe (Demske 2010: 29f.) zuständig, sondern das Morphem bzw. der Infinitivmarker -en für die Kennzeichnung von Finalität, der diachron Träger einer finalen Semantik ist (Curme 1922, Haspelmath 1989) mit synchron weiterhin modaler Semantik (Rapp/ Wöllstein 2009, 2013). Paul (1920: 290;\$330), Behaghel (1924: S.410; \$202) und ganz deutlich Curme (1922: 270) führen den reinen Infinitiv, resp. den Infinitivmarker -en auf den Akkusativ des Ziels zurück; damit war -en ursprünglich mit modaler Semantik finaler Ausprägung ausgestattet - weswegen die Bezeichnung des (reinen) Infinitivs als "Grundform" irreführend ist (Bech 1955/57: 13ff., Haspelmath 1989: 287)." 
(keine) Infinitiv-Beschränkung bei um-zu

(35) a. ${ }^{*}$ Hans ist (im Wald) sich vor Geistern fürchten.

b. Hans ist im Wald, um sich vor Geistern zu fürchten.

c. ${ }^{\star}$ Theo ist (im Bett) aufwachen.

d. Theo ist im Bett, um aufzuwachen.

Im Gegenwartsdeutschen kann das Fehlen der lexikalischen Ausdrucksmittel $(u m-z u)$ für Finalität durch intentionale Verbbedeutung und intentionale Subjekte kompensiert werden. Darüber hinaus gelten weitere Beschränkungen bei $u m$ - $z u$-Infinitiv, $z u$-Infinitiv und reinem Infinitiv je nach der Sorte des prädikativen Komplements: a) [+um+zu] ist beschränkt auf overte Realisierung des prädikativen Komplements (36), b) [-um+zu] ist ausschließlich beschränkt auf overte $\left.\mathrm{PP}_{\mathrm{LOC}}(37), \mathrm{c}\right)[-\mathrm{um}-\mathrm{zu}]$ ist ebenfalls beschränkt auf $\mathrm{PP}_{\mathrm{LOC}}$, die aber covert sein darf (38) - zusammengefasst in (39):

(36) a. Hans ist [ ${ }_{p P}$ in München], um seine politischen Ziele durchzusetzen.

b. Hans ist [NP $_{\mathrm{N}}$ Bürgermeister], um seine politischen Ziele durchzusetzen.

c. ${ }^{*}$ Irene ist $\left[{ }_{\mathrm{PP}-\mathrm{LOC}} \varnothing\right]$ um ihre Mutter zu besuchen.

(37) a. Hans ist $\left[_{\mathrm{pp}}\right.$ in München], seine politischen Ziele durchzusetzen.

b. ${ }^{*}$ Hans ist $\left[_{\mathrm{NP}}\right.$ Bürgermeister], seine politischen Ziele durchzusetzen.

c. ${ }^{*}$ Irene ist $\left[{ }_{\text {PP-LOC }} \varnothing\right]$ ihre Mutter zu besuchen.

(38) a. Hans ist [ in München], seine politischen Ziele durchsetzen.

b. ${ }^{*}$ Hans ist $\left[_{\mathrm{NP}}\right.$ Bürgermeister], seine politischen Ziele durchsetzen.

c. Irene ist $\left[_{\text {PP-LOC }} \varnothing\right]$ ihre Mutter besuchen.

(39) a. Max ist im Hof $/{ }^{\star} e_{\mathrm{LOC}}$ (um) Holz (zu) hacken.

b. Max ist im Hof $/ e_{\mathrm{LOC}} \mathrm{Holz}$ hacken.

Die grammatischen Verhältnisse widerlegen jede Ellipsenanalyse, da unter ihrer Annahme die Parameter für Grammatikalität konstant bleiben müssten. Die Konstruktion weist aber sehr wohl eine stumme Syntax auf, die als semantisches Substitut zu der Finalkonstruktion fungiert (mit den besprochenen spezifischen Beschränkungen). Damit ist FH0 bestätigt, womit der Ausgangspunkt für weitere Untersuchungen zur Syntax und Pragmatik zusammenfassend folgende Struktur bildet:

a. $\left[_{\mathrm{CP}} \mathrm{NP}_{\text {INT }} \operatorname{sein}\right.$ KOPUL.A $\left.\left(P P_{\text {LOC }}\right)\left[_{\text {FINALE-VP }} \mathrm{V}_{\text {INT-INF-1.ST }}\right]\right]$ 
Dabei ist die finale Semantik an eine - wenn auch nicht notwendig ausbuchstabierte/stumme - syntaktische Struktur geknüpft. Genau diese syntaktische Struktur stellt das Formkorrelat dar, das erst die Voraussetzung für die Absentivinterpretation bereitstellt, vgl. Wöllstein 2013: 194ff.).

\section{Ausgangspunkt für sprachvergleichende Untersuchungen}

Möchte man die oben zusammengetragenen Ergebnisse für eine sprachvergleichende Perspektive nutzen, so ist i.o.S. folgendes zu prüfen:

1. Verwendung von sein als Kopula

2. Auftreten eines (stummen) Lokationsausdrucks als prädikatives Komplement

3. Finale Semantik des Adjunkts (des Infinitivs selbst oder einer (stummen) Konjunktion/Präposition)

4. Beschränkungen bzgl. Intentionalität

Es wird im Folgenden anhand von Daten und Überlegungen zum Französischen und Italienischen gezeigt, dass kontrastiv betrachtet Deutsch in einer Kopulakonstruktion mit Adjunkt (NP + Kopula + reiner Infinitiv) einerseits das Finalitätskonzept am stärksten grammatikalisiert hat und das Lokationsprädikat, lizenziert durch mehrere Faktoren, covert bleiben kann. Französisch kann unter bestimmten Bedingungen den lexikalischen finalen Marker (Konjunktion) covert realisieren, Italienisch nie (40b, c). Die Lokationsprädikation kann nur im Deutschen und Italienischen covert realisiert werden $(40 \mathrm{a}, \mathrm{c})$. Folgt man den Überlegungen zum Deutschen, ist das dem syntaktischen Status des infinitivischen Adjunkts geschuldet, das keine Satzprojektion sein darf. Das spräche dafür, dass es sich im Französischen beim finalen Marker um ein C-Element handelt und im Italienischen um einen Marker (PART) der an den Infinitiv herantritt.
a. Dt.: $\quad\left[{ }_{\mathrm{CP}} \mathrm{NP}_{\mathrm{IN} !}\right.$
b. Fr.:
$\operatorname{sein}_{\text {KOPULA }}\left(\mathrm{PP}_{\text {LOC }}\right)$
$\left[\right.$ FINALE-VP $\left._{\text {INT-INF-1.ST }} \mathrm{V}_{\text {IN }}\right]$
c. It.:
$\left[_{\mathrm{CP}} \mathrm{NP}_{\mathrm{INT}}\right.$
être KOPULA $_{\text {a }}$
essere $_{\text {KOPULA }}$
$\mathrm{PP}_{\text {LOC }}$
$\left(\mathrm{PP}_{\text {LOC }}\right)$
$\left[\left[_{C P}\left(\mathrm{KONJ}_{\text {FINAL }}\right) \mathrm{V}_{\text {INT-INF }}\right]\right]$
[VP PART-FINAL $V_{\text {INT-INF }}$ ]

Im Einzelnen nun zu den Daten im Französischen und Italienischen. 


\subsection{Vergleich zum Italienischen ${ }^{6}$}

Betrachten wir zunächst Daten zum Italienischen unter o.g. Perspektive.

$\mathrm{Zu}$ 1.: Verwendung von sein als Kopula

essere tritt im Italienischen mit prädikativem Komplement $u$. a. eingeleitet durch $a$ auf, neben instrumental, konditional und kausal ebenso in finaler Verwendung (Skytte/Salvi 2001: 554):
Gianni è a
pescare.
Gianni ist PRÄP ${ }^{7} \quad$ fischen.

$\mathrm{Zu}$ 2.: Auftreten eines (stummen) Lokationsausdrucks als prädikatives Komplement

essere i.S.v. trovarsi (dt. befinden) oder restare (dt. bleiben) kann in Prädikativkonstruktionen ohne overten Lokationsausdruck verbleiben und wird lokal interpretiert; der durch die Präposition eingeleitete Infinitiv wird in dieser Struktur von Skytte/Salvi (2001: 535-537) als Komplement analysiert - parallel zu den Arbeiten zum sog. Absentiv (außer bei Fortmann/Wöllstein 2013, Wöllstein 2013). Natürlich finden wir bei der Analyse zum Italienischen das gleiche strukturelle Problem vor, das sich ohne das Auftreten eines regulären Komplements (NP, AP, PP) im Italienischen ganz parallel zum Deutschen ergibt, vgl. (42a) ohne prädikativen Lokalisationsausdruck und (42b) mit Lokalausdruck als Komplement des Infinitivs.

(42) a. „Erasmo come farà a esserci? A quell' ora è a lavorare.“

(CORIS, Section: NARRATRomanzi)

b. „Potrai alzare la musica quanto ti pare mentre sono a sistemare il giardino e ti permetterò di mangiare patatine croccanti al cinema, il sabato sera, senza doverti trascinare fuori nell' intervallo."

(CORIS, Section:MON2001_04)

$\mathrm{Zu}$ 3.: Finale Semantik des Infinitivs selbst oder einer (stummen) Konjunktion/ Präposition

6 Für die Diskussion, die konstruierten Daten und die Sachkenntnis zum Italienischen danke ich an dieser Stelle ganz herzlich Hagen Augustin und zum Französischen Astrid Rothe.

7 Schwarze (1995: 153) betrachtet die Infinitivkonstruktionen des Typs „finites Verb $+a+$ Infinitiv" als Verbal-, nicht als Präpositionalphrasen. Folglich bezeichnet er das verbindende $a$ hier nicht als Präposition, sondern als "Junktor“. 
Durch overtes $a$ wird eine finale Semantik eingebracht; $a$ zeichnet sich aber im Unterschied zu per, das ebenfalls eine finale (außerdem: konsekutive, konzessive und kausale) Semantik haben kann, dadurch aus, dass es in der grammatischen Struktur eine spezifische Funktion als Infinitivkomplemente einleitende Verbpartikel (bzw. Junktor bei Schwarze 1995: 153) übernimmt.

Nach Einschätzung meiner Informanten (Muttersprachler/Linguisten) kann der Lokationsausdruck bei per - anders als bei $a$ - nicht unrealisiert bleiben (43d). Das spricht dafür, dass hier kategoriale Unterschiede zu vermuten sind. Im Gegensatz zu lexikalischen können funktionale Einheiten stumm bleiben (43a) vs. (43b); das ist bei Präpositionen nicht der Fall. Die zusätzliche Weglassbarkeit von $a$ ist aus der Sicht meiner Informanten in dieser Konstruktion nicht generell gegeben, falls der Lokationsausdruck unrealisiert bleibt (43c), vgl. auch den Korpusbeleg (44).

(43) a. Gianni è (in cortile) a

b. Gianni è in cortile/(*ø) per (PRÄP ${ }_{\text {FINAL }}$ )

c. Marco è (al parco) a $\quad{ }^{*} \emptyset$

d. Marco è al parco/(*ø) per tagliare la legna.

hacken das Holz.

tagliare la legna.

prendere il sole.

prendere il sole.

(44) Giovanni è a fare il militare, dove? Ah, sí, a Bari.

CORIS, Section: NARRATRacconti)

Man beachte, dass in (43) intentionale Subjekte und Verben auftreten können aber nicht müssen (45) und (46) - und zwar dann, wenn der Lokationsausdruck overt realisiert ist (45a); die Struktur mit lexikalischem Finalitätsmarker ist bei nicht-intentionalem Subjekt und Infinitiv markiert (45b).

\section{Zu 4.: Beschränkungen bzgl. Intentionalität}

(45) a. Il vino è in cantina/(*ø) a maturare.
b.

Ill vino è in cantina
PART-FINAL

Der Wein ist im Keller

reifen.

(46) „Muoio di freddo e di paura, ma se tutti lo dicono sarà pur vero che correre come pazzi con quel gelo sopra e sotto è la cosa più bella che ti può succedere al mondo. Intanto mamma, un giorno, mentre io sono a tremare in quel bianco esilio, porta mia sorella al cinema a vedere, Katia, regina senza corona." "

(CORIS, Section: NARRATRomanzi) 


\section{Zwischenfazit:}

Die Obligatorik von $a$ (43c) macht auch die Verwendung nicht-intentionaler Subjekte und Verben im Italienischen möglich - ganz parallel. Eine Beschränkung besteht aber hinsichtlich des Lokationsausdrucks, der in Fällen nicht-intentionaler Einheiten nicht covert bleiben kann, vgl. erneut ein konstruiertes Beispiele analog zu (45):
a. L'impasto è
( ${ }^{*}$ nel frigo)
a
riposare.
Der Teig ist im Kühlschrank
PART-FINAL ruhen.
b. "L'impasto è nel frigo
per
riposare.

Neben den Überschneidungen zum Muster und den grammatischen Mitteln im Deutschen muss es einer eingehenderen empirischen Untersuchung überlassen bleiben, unter welchen Bedingungen genau a) der Lokationsausdruck entfallen kann (und dann covert bleiben kann); es scheint aber an Intentionalität geknüpft zu sein - wiederholt in (40c) vs. (40c)' und b) was in diesen Kontexten genau die Partikel $a$ (als Funktionswort) jedoch nicht die Präposition per lizenziert. Hier scheint es darüber hinaus Unterschiede zwischen den Strukturen mit it. $a$ vs. dt. $z u$ zu geben, was näher zu untersuchen wäre. Soweit zu den grammatischen/lexikalischen Mitteln im Italienischen, die Anlass zu einer Finalitätsanalyse geben, ganz analog zum Deutschen.

$$
\begin{aligned}
& \text { c. It.: }\left[\mathrm{CP}_{\mathrm{CP}} \mathrm{NP}_{\text {INT }} \text { essere }_{\text {KOPULA }}\left(\mathrm{PP}_{\text {LOC }}\right)\left[a_{\text {PART-FINAL }} \mathrm{V}_{\text {INT-INF }}\right]\right] \\
& \text { c. It.: }\left[\mathrm{CP}_{\mathrm{CP}} \mathrm{NP}\right. \text { essere } \\
& \mathrm{KP}_{\text {KOPULA }}
\end{aligned}
$$

\subsection{Vergleich zum Französischen}

\section{Zu 1.: Verwendung von sein als Kopula}

Prädikative Komplemente (Frnz.: attribut sujet, siehe Riegel et al. 1998) von être im Französischen sind - wie im Deutschen NPs (48), APs (49) und PPs ${ }^{8}$ (50). Etre ist in diesen Strukturen eine Kopula.

a. Pierre est ingénieur.

b. La chaise est un meuble.

8 Laut Riegel et al. (1998: 239) ist bei être + Lokationsausdruck von être als Lokativverb in der Bedeutung se trouver (,sich befinden') auszugehen. Hierbei handelt es sich genau um die hier untersuchte Bezugssatzstruktur (Lokationsprädikation). Ob man allerdings von der Interpretation von être in den Kontexten mit Lokationsausdruck ausgehend eine eigene lexikalische Kategorie einführen sollte, muss an anderer Stelle diskutiert werden. 
c. Le premier est l'aîné d'une famille.

(WPF11/P20.31826: Premier-né, In: Wikipedia - URL: http://fr.wikipedia. org/wiki/Premier-né: Wikipedia, 2011)

(49) a. Pierre est jeune.

b. La chaise est grande.

c. Cet indicateur est essentiel.

(WPF11/D24.86450: Détermination du temps de séchage d'un marquage routier, In: Wikipedia URL: http://fr.wikipedia.org/wiki/Détermination_ du_temps_de_séchage_d'un_marquage_routier: Wikipedia, 2011)

(50) a. Pierre est dans la chambre.

b. La chaise est dans la chambre.

c. L'horloge est dans une pièce prévue à cet effet, dans la tour de la cathédrale.

(WPF11/H09.45824: Horloge astronomique de Besançon, In: Wikipedia URL: http//fr.wikipedia.org/wiki/Horloge_astronomique_de_Besançon: Wikipedia, 2011)

Es ist im Französischen nicht möglich, être direkt mit dem Infinitiv zu verknüpfen:

(51) a. ${ }^{*}$ Pierre est travailler au restaurant. (er ist arbeiten im Restaurant)

b. $\quad{ }^{\star}$ Il est pour travailler. (er ist um (zu) arbeiten)

Die Struktur bleibt auch durch Hinzufügen eines Finalitätsmarkers wie pour ungrammatisch, siehe (51b). Allein im zusammengesetzten Vergangenheitstempus kann eine Form von être direkt mit dem Infinitiv verknüpft werden (52); für diese Struktur finden sich viele Belege (53)-(55).

(52) J'ai été le trouver. (ich bin gewesen es/ihn finden)

(53) Killy a été jouer dehors avec les voisins puis elle est venu manger je lui est donner le lait et elle a été dormir.

(Forumsbeitrag, http://forum.lixium.fr/d-4932282.html)

(54) L'employée de la sécu et l'ingénieur informaticien affirment qu'ils ont été inattentifs quelques minutes quand ils ont été acheter du sirop calmant pour leur bébé et avancent l'hypothèse selon laquelle la drogue a été placée dans leurs bagages à cet instant.

(Internetbeleg, 27.5.2013, http://www.20minutes.fr/societe/1018321-francaisemprisonnes-republique-dominicaine-homme-libere-femme-attend-proces)

(55) Et juste hier, j'ai obtenu $1.000 \$$ en argent comptant de mon frère comme un cadeau. Il a été travailler à l'étranger et je ne l'ai pas vu depuis très longtemps. Il a envoyé l'argent comme un cadeau pour moi. 
(Internetbeleg, 11.6.2013, http://www.energika.org/universelles-travailler-a02023617.htm)

In dieser Struktur hat être allerdings, laut Grevisse/Goosse (2011: 1108f.), die Bedeutung von aller; (52) entspricht also einer Kombination aus einer konjugierten Form von aller und einem Infinitiv wie in $(56)^{9}$. Grevisse/Goosse diskutieren, ob sich die Struktur mit être von der mit aller unterscheidet, besonders im $\mathrm{Zu}$ sammenhang mit der implikatierten Rückkehr des Subjekts.

(56) Je suis allé le trouver.

Etre kann Infinitive mit der Präposition $\dot{a}$ anschließen (57), jedoch handelt es sich dann um periphrastische, nichtkomplexe Strukturen mit aspektueller Progessiv-Funktion:

(57) Il est à prendre sa douche. (er ist am duschen)

Obwohl die übliche Progressiv-Struktur im Französischen mit être en train de (vgl. (58) sowie (61) und (62)) gebildet wird (vgl. Squartini 1998: 119ff., Gougenheim 1971 [1929]), finden sich für être à + Infinitiv als Progressiv zahlreiche Belege, siehe (59) und (60):

(58) Il est en train de prendre sa douche. (er ist gerade dabei zu duschen)

(59) Je m'ecarte peut être un peu mais puisqu'on est a parler vocabulaire autant définir les termes.

(WDF11/D01.94058: Discussion: Débat français sur le traité constitutionnel européen, In: Wikipedia URL: http://fr.wikipedia.org/wiki/Discussion: Débat_français_sur_le_traité_constitutionnel_européen: Wikipedia, 2011)

(60) Ah oui et puis tant qu'on est à parler de Sammy Winter, il y a aussi cette vidéo signée Fred Mortagne qui est sortie récemment. Ça se passe à La Cité de la Mode à Paris...

(http://www.dammn.fr/news/588-sammy-winter-est-pro-.html; 23.4.2014)

(61) Ils sont en train de préparer une opération pour le soir même. (WPF11/A24.60516: Achille Viadieu, In: Wikipedia - URL: http:// fr.wikipedia.org/wiki/Achille_Viadieu: Wikipedia, 2011)

(62) Pierrot et Anne se disputent. Leurs cris parviennent à leur père et à Pépé, tous deux tranquillement installés dans le salon en train de lire leur journal. 
(WPF11/L34.49914:LaFamilleCokalane(quinzièmehistoire).In:Wikipedia - URL: http://fr.wikipedia.org/wiki/La_Famille_Cokalane_(quinzième_ histoire): Wikipedia, 2011)

Die Kombination aus être à und Infinitiv kann auch modal deontisch verwendet werden. In diesen Strukturen ist das Subjekt - ganz analog zum Deutschen nicht designiert, d. h. zugrundeliegend ein strukturelles Objekt:

(63) Le devoir est à rendre demain. (die Hausaufgabe ist morgen abzugeben)

(64) Oui, effectivement tout l'article est a revoir d'autant que des parties ont etaient pompez sur des sites fachos. Aoineko.

(WDF11/J00.16695: Discussion:José Bové/archive, In: Wikipedia URL: http://fr.wikipedia.org/wiki/Discussion:José_Bové/archive: Wikipedia, 2011)

(65) En cas de doutes, la notoriété est a prouver, pas l'inverse.

(WDF11/A51.56320:Discussion:Anoë,l'AgedesRéofs/Suppression,In:Wikipedia -URL: http://fr.wikipedia.org/wiki/Discussion:Anoë,_l'Age_des_ Réofs/Suppression: Wikipedia, 2011)

Bemerkenswert ist jedoch, dass être $+\grave{a}+$ Inf. wie dt. sein $+z u$-Inf modal verwendet wird (63), was für être als Auxiliar spricht. In diesen Strukturen ist être nicht als Kopula zu werten und der über $\dot{a}$ angeschlossene Infinitiv wird nicht final interpretiert. Ganz analog zum Deutschen ist zu beobachten, dass NP-Komplemente der Kopula, wenn sie final interpretiert werden sollen, über eine overte Konjunktion wie z. B. pour (dt. um) verfügen müssen - ob intentional (66) oder nicht (67).

(66) a. *Il est ingénieur développer des machines.

b. Il est ingénieur pour développer des machines.

c. En effet ce dernier est mandataire pour défendre l'intérêt matèriel et idéal des groupes d'individus au niveau international.

(WPF11/T11.77039: Théorie des relations internationales, In: Wikipedia URL: http://fr.wikipedia.org/wiki/Théorie_des_relations_internationales: Wikipedia, 2011)

(67) a. *L'autobus est un véhicule transporter des gens.

b. L'autobus est un véhicule pour transporter des gens.

c. Le réformisme est l'instrument pour arriver à la conscience et il doit habituer la prolétariat à sa future évolution.

(WPF11/F20.27478: Filippo Turati, In: Wikipedia URL: http://fr.wikipedia. org/wiki/Filippo_Turati: Wikipedia, 2011) 


\section{Zu 2.: Auftreten eines (stummen) Lokationsausdrucks}

Es scheint sich im Französischen abzuzeichnen, dass ein stummer Lokationsausdruck - als Komplement der Kopula - nicht möglich ist (68) vs. (69).

(68) a. Marc est au parc pour prendre le soleil.

b. Alors qu'il est dans le train pour rentrer chez ses parents Johan se situe dans le même wagon qu'une jeune fille en pleure, il l'a réconforte et en vient même à lui redonner le sourire.

(WPF11/J13.87395: Johan Libéreau, In: Wikipedia - URL: http:// fr.wikipedia.org/wiki/Johan_Libéreau: Wikipedia, 2011)

c. Chemin faisant, le Maltais lui fait savoir qu'il est dans la cité pour chercher une émeraude, appelée "Clavicule de Salomon ".

(WPF11/F14.10073: Fable de Venise, In: Wikipedia - URL: http:// fr.wikipedia.org/wiki/Fable_de_Venise: Wikipedia, 2011)

(69) ${ }^{*}$ Marc est pour prendre le soleil.

\section{$\mathrm{Zu}$ 3. Finale Semantik des Infinitivs selbst oder einer (stummen) Konjunktion}

Fehlt der Finalitätsmarker, werden die Beispiele von muttersprachlichen Informanten als sehr schlecht bzw. als fraglich bewertet (70); Belege sind jedoch zu finden (71):

(70) a. ??Il est dans la cuisine ( $\sqrt{ }$ pour) travailler la pâte.

b. ??Il est sous la table ( $\sqrt{ }$ pour) chercher la bague.

c. ??Il est à la cave ( $\sqrt{ }$ pour) chercher une bouteille de vin.

d. ??Il est en ville ( $\sqrt{ }$ pour) acheter des livres.

e. ??Il est à la cave $(\sqrt{ }$ pour $)$ chercher du vin.

(71) Le temps passe vite. Passage éclair dans l'expo temporaire consacrée à Lucian Freud. Peu réceptif, passons. Quitter l'endroit puis chasser le repas dans le Marais. Bagel un peu cher, mais on est à Paris manger dans un square sur un banc. (Blogeintrag im Telegrammstilhttp://mrlo.tumblr. com/post/472186455/paris-le-debriefing)

Während die Grammatikalität der Strukturen (70) noch als marginal beurteilt wird, wird sie als gänzlich ungrammatisch beurteilt, wenn das Verb im Adjunkt nicht-intentional ist und der Finalitätsmarker pour unrealisiert bleibt. Wird der Finalitätsmarker pour realisiert, dann fällt die Bewertung der entsprechenden Beispiele etwas weniger schlecht aus: 
(72) a. *Il est sous la table ("pour) trembler.

b. ${ }^{*}$ On est à la cave ("pour) grandir.

c. ${ }^{*}$ L'autobus est sur le périphérique ("pour) transporter des gens.

Die Daten bis hierher zeigen, dass die volle Struktur [NP + être $+P P\left[_{\text {Finalsatz }}\right.$ pour + Infinitiv]] im Französischen einwandfrei grammatisch ist (66b), (70); dagegen kann im Französischen die finale Konjunktion pour - wenn überhaupt - nur bei intentionalem Subjekt und Infinitiv covert bleiben (70) und (72), siehe auch authentische Daten in (73), deren Status (u. a. nachgestellte PP) natürlich zu klären ist. Bemerkenswert ist auch hier, dass ein Bestandteil der Struktur - hier die Konjunktion und nicht wie im Italienischen der Lokationsausdruck - in Abhängigkeit von Intentionalität covert bleiben kann.

(73) a. Je me demande si elle [= Marine Le Pen, d. Verf.] n'est pas plus dangereuse que son père. la preuve c'est qu'elle est danser à vienne avec les nazis en pleine campagne présidentielle, insultant ainsi toutes les victimes du nazisme, décédées ou encore vivantes.

(Forumsbeitrag: http://www.agoravox.fr/actualites/politique/article/marinele-pen-se-fracasse-sur-le-136214)

b. Nous avions le plateau américaine qui peut être un peu sale quand on est manger en ville, il est compris dans le prix de curseurs cheeseburger avec, mal payé des frites, porc argent épargné côtelettes et poulet - est délicieuse!

(Internetbeleg, Erfahrungsbericht, http://www.tripadvisor.fr/ShowUserReviews-g950976-d1835527-r117481125-The_Highway-Plympton_South_ Australia.html)

Zusammenfassend lässt sich festhalten, dass das Französische unter der Annahme der Finalitätshypothese den stärksten Beschränkungen unterliegt. Es verbleibt auch die Klärung bzw. die Bestimmung, unter welchen Bedingungen genau der Finalitätsmarker pour covert realisiert werden kann. Ganz klar scheint aber auch hier zu sein, dass Intentionalität eine der Bedingungen ist. Französisch kann demzufolge kaum von der Vollstruktur (40b) abweichen und wenn, dann nur durch semantischen Beschränkungen (40b)'.

(40)
b. Fr.: $\left[_{C P}\right.$ NP être KOPULA $_{\text {POC }}$
$\left.\left[\operatorname{Konj}_{\text {FINAL }} V_{\text {INF }}\right]\right]$
b'. Fr.: $\left[_{C P} N_{\text {INT }}\right.$ être $e_{\text {KOPULA }} P_{\text {LOC }}\left[\left(\right.\right.$ Konj $\left.\left.\left._{\text {FINAL }}\right) V_{\text {INT-INF }}\right]\right]$ 


\section{Fazit}

Bedenkt man, dass im Deutschen die finale Semantik an eine - wenn auch nicht notwendig ausbuchstabierte/stumme - syntaktische Struktur geknüpft ist und genau diese syntaktische Struktur das Formkorrelat darstellt, das erst die Voraussetzung für die Absentivinterpretation bereitstellt (40a), so zeigt sich, dass die Möglichkeiten der Realisierung der beteiligten grammatischen Komponenten übereinzelsprachlich unterschiedlich beschränkt sind - Französisch anders als Italienisch. Im Deutschen kann der Lokationsausdruck covert bleiben - so wie der lexikalische Ausdruck für Finalität (bspw. durch um $(z u)$ ) -, es liegt aber die Beschränkung auf intentionale Verben vor. Im Französischen müssen nahezu alle Komponenten der gesamten Struktur overt ausbuchstabiert werden (40b). Unter der Intentionalitätsbeschränkung kann der Finalitätsmarker im Adjunkt covert bleiben. Der grammatische Status der Struktur muss hier empirisch überprüft werden. Im Italienischen ist ebenfalls abhängig von der Intentionalitätsbeschränkung der Lokationsausdruck in der Prädikativkonstruktion als eine Komponente covert zu realisieren; der grammatische Status dieser Struktur ist nicht zweifelhaft. Soweit spricht dies dafür, dass es lohnenswert scheint, erst recht bei den Sprachen weiter in Richtung Finalitätshypothese zu gehen, bei denen die beteiligten grammatischen Mittel in der Form wie im Deutschen vorzuliegen scheinen wie dem Ungarischen oder Norwegischen.

\section{Literatur}

Abraham W (2007). Absent arguments on the Absentive: An exercise in silent syntax. Grammatical category or just pragmatic inference? Groninger Arbeiten zur Germanistischen Linguistik 45:3-16.

Abraham W (2008). Absentive arguments on the Absentive: An exercise in silent syntax. Grammatical category or just pragmatic inference? Language Typology and Universals (STUF) 61/4:358-374.

Bech G. (1955/1957). Studien über das deutsche verbum infinitum, I-II. Kopenhagen, Munksgaard.

Behaghel O (1924). Deutsche Syntax. Eine geschichtliche Darstellung. Bd. II. Die Wortklassen und Wortformen. B. Adverbium. C. Verbum. Heidelberg, Winter (Germanische Bibliothek 1.1.10. 2), 303-372.

Curme G.O (1922). A Grammar of the German Language. Second Revised Edition [Ninth printing 1964], New York, Ungar. 
Dahl Ö (2000 Hg.). Tense and aspect in the languages of Europe. Berlin \& New York, De Gruyter.

De Groot C (2000). The absentive. In: Dahl Ö (Hg.). Tense and aspect in the languages of Europe. Berlin \& New York, De Gruyter, 693-719.

Demske $U$ (2010). Finale Marker in Infinitivkonstruktionen - Wandel und Variation. In Christen H, Patocka F \& Ziegler E (Hgs.), Struktur, Gebrauch und Wahrnehmung von Dialekt. Wien, Praesens Verlag, 29-46.

Disterheft D (1981). Remarks on the History of the Indo-European Infinitive. In Folia Linguistica Historica 2/1:3-34.

Ebert KH (2000). Progressive Markers in Germanic Languages. In Dahl Ö (Hg.), Tense and aspect in the languages of Europe. Berlin \& New York, De Gruyter, 605-653.

Fortmann Chr \& Wölstein A (2013). Zum sogenannten Absentiv. In Vogel P (Hg.), GGSG Jahrbuch 2013, demnächst.

Fortmann Chr \& Wölstein A (2014). On the so-called absentive in German. Eingereicht.

Gougenheim G (1971 [1929]). Etude sur les périphrases verbales de la langue française. Habilitationsschrift, Paris, Nizet.

Grevisse $M$ \& Goosse A (2011). Le bon usage - Grammaire française (Grevisse grammaire langue française). 15. Aufl. Brüssel, De Boeck - Duculot.

Halliday MAK (1967). Notes on transitivity and theme in English, Part 2'. Journal of Linguistics 3:199-244.

Haslinger I (2007). The Syntactic Location of Events. PhD-Thesis. LOT Dissertation Series.

Haspelmath $M$ (1989). From purposive to infinitive - a universal path of grammaticization. Folia Linguistica Historica 10.1-2:287-310.

König S (2009). „Alle sind Deutschland ... außer Fritz Eckenga - der ist einkaufen! "Der Absentiv in der deutschen Gegenwartssprache. In Winkler E (Hg.), Konstruktionelle Varianz bei Verben. OPAL-Sonderheft 4/2009:42-74.

Krause O (2002). Progressiv im Deutschen: Eine empirische Untersuchung im Kontrast mit Niederländisch und Englisch. Tübingen, Niemeyer (Reihe Linguistische Arbeiten 462).

Paul H (1920). Deutsche Grammatik. Band IV: Syntax (Zweite Hälfte). Halle, Niemeyer, 93-136. 
Rapp I \& Wöllstein A (2007). Absentiv und Adsentiv: Instanzen von Topic-Comment-Strukturen - ereignisstrukturelle und pragmatische Beschränkungen. Ms. Uni Tübingen.

Rapp I \& Wöllstein A (2009). Infinite Strukturen: selbständig, koordiniert oder subordiniert. In Ehrich V, Fortmann Chr, Reich I \& Reis M (Hgs.), Koordination und Subordination im Deutschen, Linguistische Berichte, Sonderheft 16:159-179.

Rapp I \& Wöllstein A (2013). Satzwertige $z u$-Infinitivkonstruktionen. In Meibauer J, Steinbach M \& Altmann H (Hg.), Satztypen des Deutschen. Berlin \& New York, de Gruyter, 338-355.

Renzi L, Salvi G \& Cardinaletti A (2001 Hgs.). Grande grammatica italiana di consultazione. Vol. II. Bologna: il Mulino.

Riegel M, Pellat J-Ch \& Rioul $R$ (1998). Grammaire méthodique du français. 4. Aufl. Paris, PUF.

Schwarze C (1995). Grammatik der italienischen Sprache. 2. verb. Aufl. Tübingen, Niemeyer.

Squartini M (1998). Verbal periphrases in Romance: aspect, actionality, and grammaticalization. Berlin, De Gruyter.

Skytte G \& Salvi G (2001). Frasi subordinate all'infinito. In Renzi L, Salvi G \& Cardinaletti A (Hgs.). Grande grammatica italiana di consultazione. Vol. II. Bologna, il Mulino, 483-569.

Vogel PM (2007). „Anna ist essen!“ Neue Überlegungen zum Absentiv in den europäischen Sprachen mit einem Exkurs zum Deutschen. In Geist L \& Rothstein B (Hgs.), Kopulaverben und Kopulasätze: Intersprachliche und Intrasprachliche Aspekte. Tübingen, Niemeyer, 253-284.

Vogel P (2009). Absentiv. In Hentschel E \& Vogel P (Hgs.), Deutsche Morphologie. Berlin, de Gruyter.

Wöllstein A (2005). Die Verlaufsform ist am kommen. Habilvortrag Universität Köln.

Wöllstein A (2007). Einordnung der Form am + reiner Infinitiv in das Bechsche System der Status. Ms. Bergische Universität Wuppertal.

Wöllstein A (2013). Aspekte des Absentivs. In Härtl H (Hg.), Interfaces of Morphology. Berlin, Akademie-Verlag.

Zifonun G, Hoffmann L \& Strecker B (1997 Hgs.), Grammatik der deutschen Sprache. Berlin \& New York, de Gruyter (= IdS-Grammatik). 